\title{
A deep optical/near-infrared catalogue of Serpens ${ }^{\star}$
}

\author{
L. Spezzi ${ }^{1}$, B. Merín ${ }^{2}$, I. Oliveira ${ }^{3,4}$, E. F. van Dishoeck ${ }^{4,5}$, and J. M. Brown ${ }^{5}$ \\ ${ }^{1}$ Research and Scientific Support Department, European Space Agency (ESA-ESTEC), PO Box 299, 2200 AG Noordwijk, \\ The Netherlands \\ e-mail: 1spezzi@rssd.esa.int \\ 2 European Space Astronomy Center, European Space Agency (ESA-ESAC), PO Box Apdo. de correos 78, \\ 28691 Villanueva de la Cañada, Madrid, Spain \\ 3 California Institute of Technology, Division for Geological and Planetary Sciences, MS 150-21, Pasadena, CA 91125, USA \\ ${ }^{4}$ Leiden Observatory, Leiden University, PO Box 9513, 2300 RA Leiden, The Netherlands \\ 5 Max Planck Institut für Extraterrestrische Physik, Giessenbachstrasse 1, 85748 Garching, Germany
}

Received 23 December 2009 / Accepted 8 February 2010

\section{ABSTRACT}

\begin{abstract}
We present a deep optical/near-infrared imaging survey of the Serpens molecular cloud. This survey constitutes the complementary optical data to the Spitzer "Core To Disk" (c2d) Legacy survey in this cloud. The survey was conducted using the wide field camera at the Isaac Newton Telescope. About 0.96 square degrees were imaged in the $R$ and $Z$ filters, covering the entire region where most of the young stellar objects identified by the c2d survey are located. The 26524 point-like sources were detected in both $R$ and $Z$ bands down to $R \approx 24.5 \mathrm{mag}$ and $Z \approx 23 \mathrm{mag}$ with a signal-to-noise ratio better than 3 . The $95 \%$ completeness limit of our catalogue corresponds to $0.04 M_{\odot}$ for members of the Serpens star-forming region (age $2 \mathrm{Myr}$ and distance $260 \mathrm{pc}$ ) in the absence of extinction. Adopting the typical extinction of the observed area $\left(\mathrm{A}_{V} \approx 7 \mathrm{mag}\right.$ ), we estimate a $95 \%$ completeness level down to $M \approx 0.1 M_{\odot}$. The astrometric accuracy of our catalogue is 0.4 arcsec with respect to the 2MASS catalogue. Our final catalogue contains J2000 celestial coordinates, magnitudes in the $R$ and $Z$ bands calibrated to the SDSS photometric system and, where possible, $J H K_{S}$ magnitudes from 2MASS for sources in 0.96 square degrees in the direction of Serpens. This data product has already been used within the frame of the c2d Spitzer Legacy Project analysis in Serpens to study the star/disk formation and evolution in this cloud. Here we use it to obtain new indications of the disk-less population in Serpens.
\end{abstract}

Key words. catalogs - stars: formation - stars: low-mass - stars: brown dwarfs - ISM: clouds - ISM: individual objects: Serpens

\section{Introduction}

The Spitzer Legacy Survey "Molecular Cores to Planet Forming Disks" (c2d; Evans et al. 2003) has offered a singular opportunity for a major advance in the study of star and planet formation. Thanks to its sensitivity and wavelength coverage, Spitzer allowed us to address for the first time long-standing challenges such as disk formation and dispersal, the physical and chemical evolution of the circumstellar material and, in particular, to probe the inner planet-forming region of disks on the basis of statistically significant samples (see, e.g., Lada et al. 2006).

One of the star-forming regions included in the c2d survey is the Serpens molecular cloud. Because of its proximity (260 pc; Straizys et al. 1996) and young age (2-6 Myr; Oliveira et al. 2009), this cloud is particularly well-suited to studies of very young low-mass stars and substellar objects. The c2d survey in Serpens has provided evidence of sequential star formation in this cloud progressing from SW to NE and culminating in the main Serpens Core with its cluster of Class 0 objects (Kaas et al. 2004; Harvey et al. 2007); moreover, the surface density of young stars in this region is much higher, by a factor of 10-100, than that of the other star-forming regions mapped by the $\mathrm{c} 2 \mathrm{~d}$

* Catalogue (in VizieR) is only available in electronic form at the CDS via anonymous ftp to

http://cdsarc.u-strasbg.fr/viz-bin/Cat team (Evans et al. 2009) and includes $22 \%$ of the c2d sources classified as "transitional" disks. This makes Serpens the best region for obtaining a complete, well-defined sample of multiwavelength observations of young stars and substellar objects in a possible evolutionary sequence to build up a "template" sample for the study of disk evolution up to a few Myr within a single, small, well-defined region. To this aim, the $\mathrm{c} 2 \mathrm{~d}$ Team has conducted several surveys, from X-ray to millimetre wavelengths, and spectroscopic follow-ups of the newly discovered population of young stars in Serpens, making this cloud only the third star-forming region after Taurus and IC 348 for which such an unbiased dataset exists (Goodman 2004; Harvey et al. 2007; Enoch et al. 2007; Oliveira et al. 2009, 2010, in prep.).

In this paper we present the optical/near-infrared (NIR) imaging data collected within the frame of the c2d survey in Serpens. These data represent one of the critical ingredients to anchor the studies of envelopes and disks to the properties of the central stars, including those without infrared excess. Indeed, deep XMM-Newton data of the same field revealed a sample of new sources, mostly candidate weak-line T Tauri stars (WTTSs), half of which have no counterpart in other catalogues (Brown et al. 2010, in prep.). Moreover, Comeròn et al. (2009) recently reported on a large-scale optical survey of the Lupus star-forming complex, which, in combination with NIR data from the 2MASS catalogue, unveiled a large population of stars and brown dwarfs (BDs) that have lost their inner disks on a 
Table 1. Journal of the observations.

\begin{tabular}{cccccc}
\hline \hline $\begin{array}{c}\text { Field } \\
\text { (RA, Dec) }\end{array}$ & $\begin{array}{c}\text { Date } \\
(\mathrm{d} / \mathrm{m} / \mathrm{y})\end{array}$ & Filter & $\begin{array}{c}T_{\exp } \\
(\mathrm{min})\end{array}$ & $\begin{array}{c}\text { Seeing } \\
\left({ }^{\prime \prime}\right)\end{array}$ & Air Mass \\
\hline Serpens_1 & $13 / 05 / 08$ & $R$ & $120 \times 4$ & 1.5 & 1.50 \\
$(18: 28: 43,+00: 24: 46)$ & $17 / 05 / 08$ & $R$ & $10 \times 1$ & 2.0 & 1.14 \\
& $15 / 05 / 08$ & $Z$ & $120 \times 9$ & 1.5 & 1.17 \\
& $17 / 05 / 08$ & $Z$ & $10 \times 1$ & 2.1 & 1.14 \\
\hline Serpens_2 & $14 / 05 / 08$ & $R$ & $120 \times 9$ & 1.6 & 1.15 \\
$(18: 28: 57,+01: 02: 54)$ & $17 / 05 / 08$ & $R$ & $10 \times 1$ & 2.0 & 1.15 \\
& $15 / 05 / 08$ & $Z$ & $120 \times 4$ & 1.5 & 1.16 \\
& $17 / 05 / 08$ & $Z$ & $10 \times 1$ & 1.5 & 1.15 \\
\hline Serpens_3 & $14 / 05 / 08$ & $R$ & $120 \times 9$ & 1.6 & 1.15 \\
$(18: 28: 36,-00: 09: 51)$ & $17 / 05 / 08$ & $R$ & $10 \times 1$ & 1.6 & 1.15 \\
& $17 / 05 / 08$ & $Z$ & $10 \times 1$ & 2.3 & 1.15 \\
\hline L 104 & $13 / 05 / 08$ & $R$ & $2 \times 1$ & 1.2 & 1.22 \\
$(12: 41: 68,-00: 34: 11)$ & $13 / 05 / 08$ & $Z$ & $5 \times 1$ & 1.3 & 1.19 \\
\hline
\end{tabular}

Notes. ${ }^{(\dagger)}$ Standard star field.

time-scale of a few Myr or less. This discovery stresses the important unknowns that persistin the observational characterisation of young very low-mass objects and in the time-scales and mechanisms for disk dissipation.

The outline of this paper is as follows: in Sects. 2 and 3 we describe the observations and data reduction procedure, with particular emphasis on the photometric completeness and astrometric accuracy of the final optical/NIR catalogue in Sect. 4 we discuss the use of this catalogue within the frame of the c2d Team's analysis in Serpens and investigate its disk-less population. Our conclusions are drawn in Sect. 5.

\section{Observations}

The imaging observations presented in this work were carried out from 13 to 17 May 2008 using the Wide Field Camera (WFC) at the $2.5 \mathrm{~m}$ Isaac Newton Telescope (INT), which is located at the Roque de Los Muchachos Observatory (La Palma, Spain). The data were collected by Ignas Snellen as part of an observing practicum for Leiden University astronomy students.

The WFC is a four-chip mosaic of thinned AR coated EEV $4 \mathrm{~K} \times 2 \mathrm{~K}$ devices. Each $\mathrm{CCD}$ has an useful imaging area of $2048 \times 4100$ pixels with a pixel scale of 0.333 arcsec/pixel, covering a total field of view $34 \times 34$ arcmin with small gaps of $\sim 20^{\prime \prime}$ between adjacent chips. The average CCD read-out noise and gain are $7 \mathrm{e}^{-}$and $2.8 \mathrm{e}^{-} / \mathrm{ADU}$, respectively.

A total of 3 fields were observed in the direction of Serpens $\left(\mathrm{RA}=18^{\mathrm{h}} 29^{\mathrm{m}} 49^{\mathrm{s}}\right.$, Dec $\left.=+01^{\mathrm{d}} 14^{\mathrm{m}} 48^{\mathrm{s}}\right)$, covering the entire region where most of the young stellar objects (YSOs) identified by the Spitzer c2d survey are located (Fig. 1). Images were obtained through the $R\left(\lambda_{\mathrm{c}}=6260 \AA, F W H M=1380 \AA\right)$ and $Z\left(\lambda_{\mathrm{c}}=9100 \AA, F W H M=1370 \AA\right)$ filters approximately covering the Sloan Digital Sky Survey (SDSS) $r^{\prime}$ and $z^{\prime}$ bands (Fukugita et al. 1996). The total sky-area observed in each filter is 0.96 square degrees. Each observation in each filter was split into several individual exposures (ditherings), shifting the telescope pointing by $\sim 1$ arcmin between consecutive exposures; this allows us to cover the gaps between the CCDs and avoid saturation of bright sources in the field. The exposure time for each dithering was $120 \mathrm{~s}$ in both the $R$ and $Z$ bands. In order to recover the photometry for bright sources saturated in these long-time exposures, a series of short-time exposures (i.e. $10 \mathrm{~s}$ per dithering in both filters) of the same sky-area was also performed. The summary of these observations is reported in Table 1. A standard Landolt field was also observed in both filters for absolute flux calibration purposes (Sect. 3.3).

\section{Data reduction}

The raw images were processed using the IRAF ${ }^{1}$ mscred package and a number of scripts ad hoc developed both under IRAF and under IDL ${ }^{2}$. We followed the steps for the WFC data reduction pipeline (Irwin \& Lewis 2001) developed by the Cambridge Astronomical Survey Unit (CASU), responsible for the processing and archiving of the dataset from the INT Wide Field Survey (WFS; McMahon et al. 2001).

\subsection{Pre-reduction}

Since the bias level tends to vary, the images were first corrected for overscan and trimmed using the bias and trim sections as specified in the FITS headers. For each night in which observations for our program were performed, twilight flat frames were then combined to obtain the night master flat, which was then used to correct the science images. The corrected images are essentially linear to $\sim 2 \%$ over the full range. Bad pixels and partial columns have been replaced using the bad pixel mask files available from the WFS homepage ${ }^{3}$.

The $Z$-band images suffer from significant sky fringes. In order to remove them, we subtracted from each $Z$-band image the fringing pattern frame available from the WFS homepage scaled by a specific factor to account for the amplitude of the fringes in the individual science frames.

\subsection{Astrometry and co-addition of images}

The astrometric calibration and relative flux scaling between ditherings were obtained using the c-version of ASTROMETRIX $^{4}$ (M. Radovich, private comm.). This tool performs a global astrometric solution that takes overlapping sources falling on adjacent CCDs in different ditherings into account. For each pointing, the astrometric solution was computed using the USNO-B1.0 catalogue (Monet et al. 2003) as a reference. Within the global astrometry process, the astrometric solution was constrained for each CCD by both the positions from the USNO-B1.0 catalogue and those from overlapping sources in all the other CCDs.

The co-addition of the dithered images for a given filter and pointing was performed using the SWARP tool (Bertin 2008). The final stacked image is a $6 k \times 6 k$ frame where each pixel value is the median flux of the co-added ditherings normalised to the total exposure time and relative to the airmass and atmospheric transparency of the first frame in the dithering set. The absolute astrometric precision of our images is about 0.4 arcsec, slightly lower than RMS accuracy of the USNO-B1.0 catalogue $(0.2$ arcsec); the astrometric precision has been also confirmed by a cross-check with the 2MASS point-source catalogue (Fig. 2). The internal RMS, computed from overlapping sources in different exposures, is within 0.05 arcsec, indicating the good performance of ASTROMETRIX.

\footnotetext{
${ }^{1}$ IRAF is distributed by NOAO, which is operated by the Association of Universities for Research in Astronomy, Inc., under contract to the National Science Foundation.

2 Interactive Data Language.

3 http://www.ast.cam.ac.uk/ wfcsur/technical/pipeline/

${ }^{4}$ See also: http://www. na. astro.it/ radovich/wifix.htm
} 


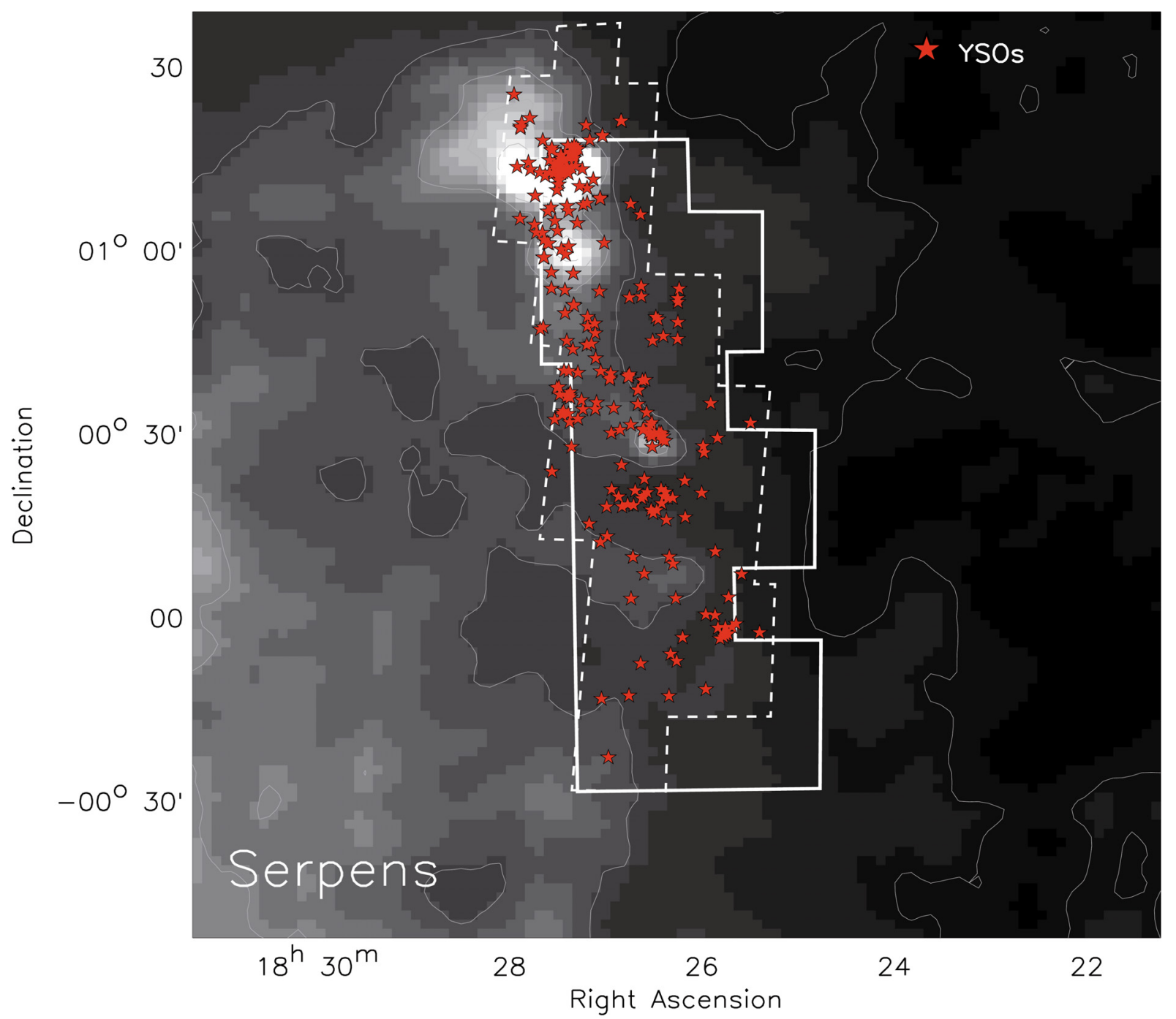

Fig. 1. IRAS $100 \mu \mathrm{m}$ dust emission map of the Serpens dark cloud. The contours, from 55 to $190 \mathrm{MJy} \times \mathrm{sr}^{-1}$ in steps of $9 \mathrm{MJy} \times \mathrm{sr}^{-1}$, are also drawn (white lines). The continuous line defines the area covered by the three INT+WFC pointings, while the dashed line is the area observed by Spitzer as part of the c2d Survey. The stars are the YSO candidates identified by the Spitzer c2d Survey.

\subsection{Photometric calibration}

Instrumental magnitudes were reported to the standard SDSS photometric system (Fukugita et al. 1996). To this aim, the Stetson's standard star field L 104 (Stetson 2000) was observed in the $R Z$ filters. By using the IRAF package photcal, we first performed the aperture photometry for the standard stars, obtaining their instrumental magnitudes $\left(r_{0}\right.$ and $\left.z_{0}\right)$ corrected for atmospheric extinction and normalised to the exposure time. Then, the transformation coefficients, namely zero point $(Z P)$ and colour term $(c)$, from the WFC-INT system to the SDSS standard system were determined by a linear fitting of the following equations:

$r^{\prime}=r_{0}+c_{R} \cdot\left(r_{0}-z_{0}\right)+Z P_{R}$

$z^{\prime}=z_{0}+c_{Z} \cdot\left(r_{0}-z_{0}\right)+Z P_{Z}$

where $r^{\prime}$ and $z^{\prime}$ are the standard magnitudes of Landolt's stars in the SDSS system.

The mean transformation coefficients determined in our observing run are reported in Table 2.

Using these coefficients, instrumental magnitudes (see Sect. 3.4) for the observed sources in Serpens have been converted to the SDSS photometric system, which is nearly an AB
Table 2. Mean photometric calibration coefficients for our WFC-INT observing run.

\begin{tabular}{cccc}
\hline \hline Filter & $K^{\dagger}$ & $Z P$ & $c$ \\
\hline$R$ & 0.0734 & $25.30 \pm 0.03$ & $-0.033 \pm 0.024$ \\
$Z$ & 0.0103 & $23.52 \pm 0.03$ & $0.021 \pm 0.022$ \\
\hline
\end{tabular}

Notes. ${ }^{(\dagger)} K=$ mean atmospheric extinction coefficients for La Palma. ${ }^{(\ddagger)} Z P=$ photometric zero point (see Eq. $\left.(2)\right) .{ }^{(*)} c=$ photometric colour term (see Eq. (2)).

system. Thus, magnitudes in our catalogue can be turned into flux densities using the correction from SDSS zero points to $\mathrm{AB}$ zero points and the $\mathrm{AB}$ zero point flux density ${ }^{5}$ :

$$
\begin{aligned}
& \text { correction } Z P_{R}=0 \mathrm{mag} \\
& F_{r^{\prime}}^{0}=2.7769 \mathrm{E}-12 \mathrm{~W} \cdot \mathrm{cm}^{-2} \cdot \mu \mathrm{m}^{-1} \\
& \text { correction } Z P_{Z}=0.02 \mathrm{mag} \\
& F_{z^{\prime}}^{0}=1.3153 E-12 \mathrm{~W} \cdot \mathrm{cm}^{-2} \cdot \mu \mathrm{m}^{-1}
\end{aligned}
$$

${ }_{5}$ See http://www.sdss.org/dr6/algorithms/fluxcal.html. 

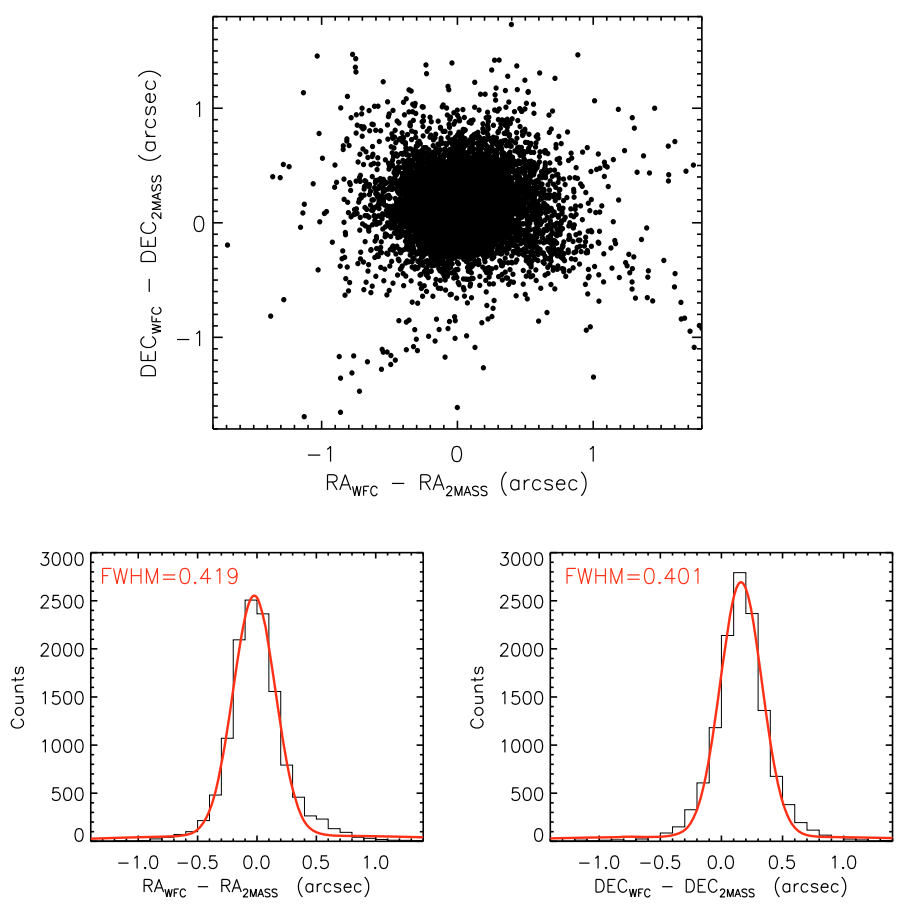

Fig. 2. Residuals of the coordinates obtained by us for the sources in the surveyed area in Serpens with respect to those from the 2MASS catalogue.

\subsection{The catalogue extraction}

The source extraction and photometry from each stacked image in each filter were performed by using the 2.5 version of the SExtractor tool by Bertin \& Arnouts (1996). SExtractor exploits the aperture photometry technique, which is the faster and best approach for uncrowded fields such as the one considered here. In particular, we adopted the SExtractor adaptive aperture magnitudes ( $\left.m a g_{\text {auto }}\right)$, which are estimated from a flexible elliptical aperture around each detected object; this method is expected to give the most precise magnitudes for very faint objects which may appear extended. The detection threshold was set in order to select all the sources having a signal-to-noise ratio $(\sigma)$ greater than 3 . The background is locally estimated from a ring shaped region surrounding the star.

The output catalogues contain for each source an identification number, right ascension and declination in degree at J2000, instrumental magnitudes and relative errors, and two SExtractor morphological parameters, namely the extraction flag (FLAGS) and the isophotal area above the threshold (ISOAREA_IMAGE). These last parameters were used to clean the catalogues from spurious detections, such as cosmic ray hits, bad pixels, saturated or truncated sources too close to the image boundaries, etc.

In Fig. 3 we show as an example the internal photometric errors of all the point-like sources detected in our long-time exposures plotted against the magnitude for the $R Z$ filters; the relative exponential fits are over-plotted. Table 3 summarises the saturation limit and the limiting magnitude achieved at the $10 \sigma$, $5 \sigma$ and $3 \sigma$ level in each filter for both the long time exposures and the short ones.

Our final catalogue contains celestial coordinates (RA and Dec at J2000) and $R Z$ photometry for each point-like object detected in our survey above the $3 \sigma$ level. Each entry in the catalogue was further complemented with near-infrared $\mathrm{JHK}_{S}$ photometry by cross-matching it with the 2MASS Point Source
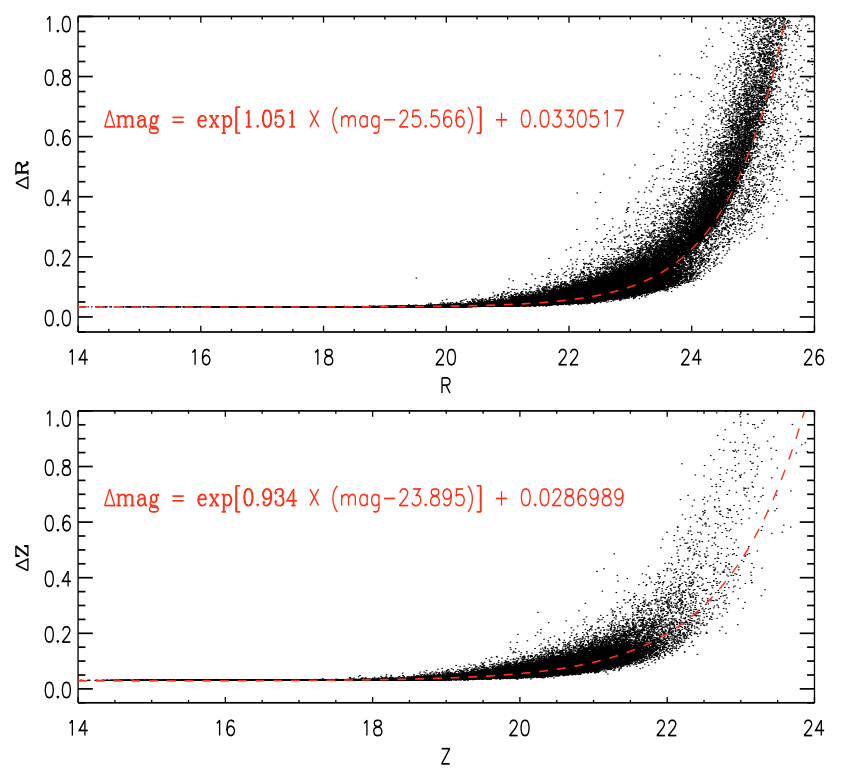

Fig. 3. Photometric errors versus magnitudes and relative exponential fits for all the point-like sources detected in the 0.96 square degrees area surveyed in Serpens. Photometry is from our long-time exposures (Sect. 2).

Catalog (limiting magnitude $K_{S} \approx 15.5$; Skrutskie et al. 2006). A matching radius of 2 arcsec was defined, which appears to be sufficient given that more than $90 \%$ of the matches corresponded to differences between the 2MASS positions and those determined by us of less than 1 arcsec. When more than one 2MASS source was found within the 2 arcsec circle, the one closer to the position derived from our observations was chosen as the counterpart.

The full catalogue is downloadable from http: //cdsarc.u-strasbg.fr/viz-bin/Cat

\subsection{Completeness}

The completeness of our catalogue was estimated in the standard way by inserting artificial stars into the images and recovering them using the same extraction procedure as for the real objects (Sect. 3.4); the fraction of recovered artificial objects provides a measure of the completeness.

We used the DAOPHOT II standalone package to perform the exercise (Stetson 1987). We first use the PSF task to extract from the $R$ and $Z$ mosaics the relative PSF models. Then we inserted in each mosaic 1000 artificial sources using the addstar task; this number should not alter the crowding statistics in the images significantly. The profile for the artificial sources was generated by using the relative PSF model, while their positions are randomly distributed over the entire area of the mosaic and their magnitudes range uniformly between the detection and the saturation limits in the relative filter (see Table 3).

Figure 4 shows the fraction of recovered artificial objects as a function of magnitude from both the deep and shallow mosaics for the $R$ and $Z$ filters. The corresponding magnitude limits at 95\% completeness level $(C=95 \%)$ are reported in Table 3 .

In the absence of extinction $\left(A_{V}=0\right)$, assuming a typical age for Serpens members of 2-6 Myr (Oliveira et al. 2009), a distance of 260 pc (Straizys et al. 1996) and using the theoretical isochrones and evolutionary tracks by Baraffe et al. (1998) and Chabrier et al. (2000) and the completeness limits from 
Table 3. Number of stellar sources $\left(N_{\mathrm{S}}\right)$ detected in both filters above the $3 \sigma$ level and limiting magnitudes in each filter at $10 \sigma, 5 \sigma, 3 \sigma$ and $95 \%$ completeness level.

\begin{tabular}{lccccccc}
\hline \hline & $N_{\mathrm{S}}$ & Filter & Mag Sat. & Mag 10 $\sigma$ & Mag 5 $\sigma$ & Mag 3 $\sigma$ & Mag $(C=95 \%)$ \\
\hline Long Time Exposures & 26524 & $R$ & 16.00 & 22.90 & 23.87 & 24.49 & 22.30 \\
& & $Z$ & 14.50 & 21.12 & 22.11 & 22.73 & 19.20 \\
\hline Short Time Exposures & \multirow{2}{*}{3709} & $R$ & 12.50 & 19.82 & 20.84 & 21.50 & 18.30 \\
& & $Z$ & 10.20 & 18.61 & 19.41 & 19.91 & 16.10 \\
\hline
\end{tabular}

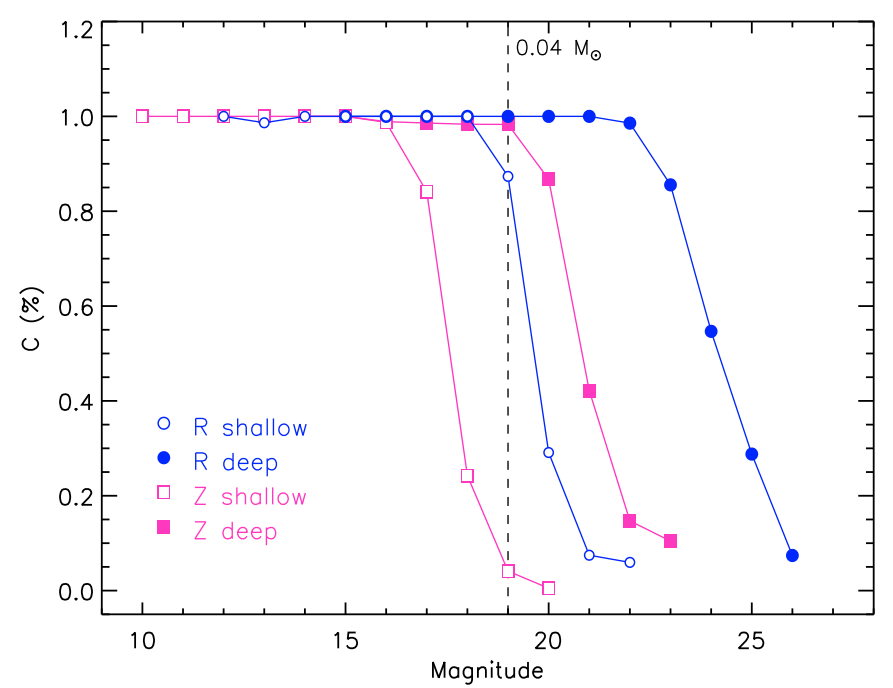

Fig. 4. Completeness $(C)$ plot for extraction of artificial stars from our "deep" and "shallow" mosaics for the $R$ and $Z$ bands.

Table 3, our optical survey would be complete down to $0.04 M_{\odot}$ at the $95 \%$ level, i.e. well below the hydrogen burning limit $\left(\sim 0.08 M_{\odot}\right)$. However, as shown by Harvey et al. (2007) in their Fig. 2, the visual extinction toward the area observed in Serpens has a typical value of $A_{V} \approx 7 \mathrm{mag}$. Adopting this extinction, we estimate a $95 \%$ completeness level down to $M \approx 0.1 M_{\odot}$,

\section{On the disk-less population in Serpens}

The observations presented in this paper are part of the $c 2 d$ complementary work in Serpens. The optical/NIR catalogue described in Sect. 3.4 has been merged with the existing X-ray to millimetre wavelengths observations collected by the c2d Team for Serpens (Goodman 2004; Harvey et al. 2007; Enoch et al. 2007; Oliveira et al. 2009, 2010, in prep.), in order to construct complete spectral energy distributions (SEDs) of the YSOs in the observed fields. This dataset allows us to anchor disk properties of the Serpens YSO sample to the mass, age, and evolutionary status of the central object, which is a critical point in the studies of envelopes/disks formation and evolution (see, e.g., Kundurthy et al. 2006; Meyer 2009; Pascucci et al. 2009). A number of papers based on the use of the $\mathrm{c} 2 \mathrm{~d}$ extensive dataset for Serpens and presenting results on disk evolution are in preparation.

As mentioned in Sect. 1, Comeròn et al. (2009) have recently identified a large population of young members of the Lupus dark cloud complex which seems to have lost their inner disks on a time-scale of a few Myr or less. This discovery poses the important question of whether the existence of such disk-less young stars is the outcome of specific star-forming conditions in Lupus or similar populations exist in other regions.

XMM-Newton data obtained in April 2007 and April 2008 of the same field observed by the c $2 \mathrm{~d}$ survey in Serpens revealed a sample of new sources, half of which have no counterpart in the c2d catalogue and are mostly candidate WTTSs (Brown et al. 2010 , in prep.). For $\sim 44 \%$ of the objects in this sample, we find optical and NIR colours consistent with those of young objects with no prominent IR excess. Indeed, they occupy in the $Z$ vs. $R-Z$ (Fig. 5, upper panel) the same locus as the YSOs identified by the c2d survey (Harvey et al. 2007), which corresponds to an age between 1 and $10 \mathrm{Myr}$ according to pre-main sequence (PMS) isochrones by Baraffe et al. (1998) and Chabrier et al. (2000) and is consistent with the ages of the c2d YSOs found by Oliveira et al. (2009). The isochrones have been matched to the SDSS photometric system following the procedure described in Appendix B by Spezzi et al. (2007). On the $J-H$ vs. $H-K$ diagram the c2d YSOs follow, as expected, the dwarf stars locus with many of them presenting IR excess with respect to this locus; the X-ray sources mainly concentrate on the field dwarfs locus, as expected for objects with no disk. For the remaining objects in the X-ray source sample we could not perform this analysis because they are either saturated or not detected in our optical/NIR survey, while a few of them are out of the observed field.

To further investigate the disk-less population in Serpens, we tried to apply to our dataset the novel $S$-parameter method by Comeròn et al. (2009), which allows the identification of possible young stars and substellar objects based on their optical/NIR photospheric fluxes, independently of the display of signposts of youth, such as IR excess emission or strong $\mathrm{H} \alpha$ emission. The $S$-parameter method simultaneously estimates the visual extinction $\left(A_{V}\right)$, effective temperature $\left(T_{\text {eff }}\right)$ and a wavelength-independent scaling factor $(S)$, containing the dependency on the actual distance and radius of the star, by fitting a grid of stellar photosphere models to the observed SED. Using the Galactic star counts model by Wainscoat et al. (1992), Comeròn et al. (2009) demonstrated that members of nearby young star forming regions at a given temperature $(<4000 \mathrm{~K})$ and within a restricted set of ages $(<20 \mathrm{Myr})$ are characterised by values of $S$ virtually unreachable by non-members of similar temperature, both foreground dwarfs and background giants. This method is expected to work reasonably well for the detection of cool populations associated to star-forming regions located in the $\sim 100-300$ pc distance range from the Sun (see Sect. 5 by Comeròn et al. 2009) and, indeed, it has been successfully applied to a number of nearby star forming regions: Lupus (Comeròn et al. 2009), Cha I (Lopèz Martì, private communication), Cr A (Lòpez Martì et al. 2010) and Cha II, where it provides the same results as in Spezzi et al. (2007). We applied the $S$-parameter method to the optical/NIR dataset for Serpens; we find that in this case the $S$-parameter histogram at any temperature range shows no clear separation between the member candidates and the background/foreground contaminants and, as a consequence, the method produces an unreliable sample of member candidates, which is also not compatible with the XMM-detected sources in the same area of the sky. There are recent indications (see Sect. 2.3 by Merìn et al. 2008) that Galactic 

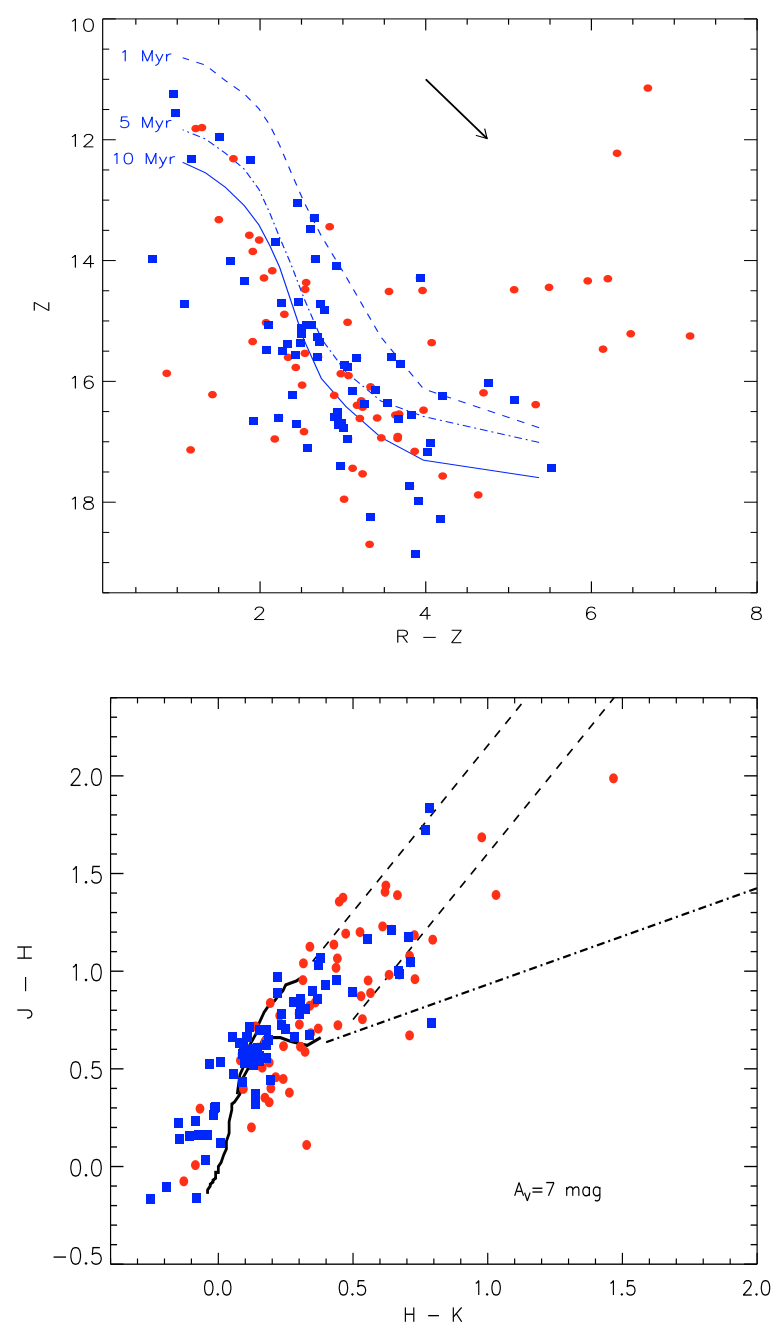

Fig. $5 . Z$ vs. $R-Z$ and $J-H$ vs. $H-K$ diagrams for the YSOs in Serpens selected by the c2d survey (circles) and the optical/NIR counterparts of the X-ray sources identified by (Brown et al. 2010, in prep.) (squares). Upper panel: the lines are the PMS isochrones by Baraffe et al. (1998) and Chabrier et al. (2000) transformed by us into the SDSS photometric system. The arrow represents $A_{V}=2$ reddening vector (Weingartner \& Draine 2001). Lower panel: the solid curve shows the relation between the colour indices for main sequence stars (lower branch) and giants (upper branch), together with the relative reddening bands (dashed lines). The dash-dot line is the T Tauri star locus by Meyer et al. (1997). The object magnitudes are derreddened using the typical extinction in the observed area $\left(A_{V} \approx 7 \mathrm{mag}\right)$ and the extinction curve by Weingartner \& Draine (2001).

models may fail at reproducing stellar counts at low galactic latitude and, indeed, Serpens lies very close to the galactic plane $(b \approx+5.4 \mathrm{deg}$ ). Moreover, Serpens is located at $260 \mathrm{pc}$ from the Sun, i.e. at the limit of the validity range of the $S$-parameter method. Since all the other clouds on which the method has been tested are closer to the Sun and have higher galactic latitude than Serpens, we conclude that the unlucky combination of distance and position of this cloud prevents the $S$-parameter method from giving useful results.

\section{Conclusions}

We presented an optical/NIR catalogue ( $R$ and $Z$ filters) of 26524 point-like sources in 0.96 square degrees in the direction of Serpens down to $R \approx 25$. These data were collected using the WFC camera at the INT within the frame of the Spitzer c2d survey in Serpens. The catalogue was also complemented with $J H K_{S}$ photometry from 2MASS and has been merged with the existing X-ray to millimetre wavelengths observations collected by the $\mathrm{c} 2 \mathrm{~d}$ Team for Serpens to study the envelop/disk formation and evolution and its dependency on the stellar properties. A number of paper based on this comprehensive catalogue are now published or in preparation (Merìn et al. 2010a,b, in prep.; Oliveira et al. 2010, in prep.; Brown et al. 2010, in prep.).

In this paper we used the optical/NIR catalogue to investigate the disk-less population in Serpens. Because of the distance and low galactic latitude of this cloud, the $S$-parameters method by Comeròn et al. (2009), suitable for the identification of young objects independently of any signature of youth, fails in distinguishing young cloud members from field contaminants. However, a sample of new WTTS candidates has been identified in Serpens on the basis of XMM-Newton observations. Our optical/NIR photometry suggests a very young age ( $\lesssim 10 \mathrm{Myr})$ and no NIR excess emission for about $44 \%$ of them, supporting their WTTS nature.

Acknowledgements. This publication makes use of data products from the Two Micron All Sky Survey, which is a joint project of the University of Massachusetts and the Infrared Processing and Analysis Center/California Institute of Technology, funded by NASA and the National Science Foundation. We also acknowledge extensive use of the SIMBAD database, operated at CDS Strasbourg. We thank Ignas Snellen and the students from Leiden Observatory for taking these INT data in service time. We thank Mario Radvich for the concession of ASTROMETRIX. We are also grateful to F. Comerón, J.M. Alcalá, H. Bouy, B. Lopéz Martí, R. Jayawardhana and the Spitzer c2d Team for useful discussions and suggestions. We are also grateful to many others, in particular to Salvatore Spezzi.

\section{References}

Baraffe, I., Chabrier, G., Allard, F., \& Hauschildt, P. H. 1998, A\&A, 337, 403 Bertin, E., \& Arnouts, S. 1996, A\&AS, 117, 393

Bertin, E. 2007, SWARP v2.17.0 User's guide

Chabrier, G., Baraffe, I., Allard, F., \& Hauschildt, P. 2000, ApJ, 542, 464

Comerón, F., Spezzi, L., \& Lòpez Martì, B. 2009, A\&A, 500, 1045

Enoch, M. L., Glenn, J., Evans, N. J. II, et al. 2007, ApJ, 666, 982

Evans, N. J. II, Allen, L. E., Blake G. A., et al. 2003, PASP, 115, 965

Evans, N. J. II, Dunham, M. M., Jorgensen, J. K., et al. 2009, ApJS, 181, 321

Fukugita, M., Ichikawa, T., Gunn, J. E., et al. 1996, AJ, 111, 1748

Goodman, A. A. 2004, ASPC, 323, 171

Harvey, P., Merń, B., Huard, T. L., et al. 2007, ApJ, 663, 1149

Irwin, M., \& Lewis, J. 2001, New AR, 45, 105

Kaas, A. A., Olofsson, G., Bontemps, S., et al. 2004, A\&A, 421, 623

Kundurthy, P., Meyer, M. R., Robberto, M., et al. 2006, AJ, 132, 2469

Lada, C. J., Muench, A. A., Luhman, K. L., et al. 2006, AJ, 131, 1574

Lòpez Martí, B., Spezzi, L., Merín, B., et al. 2010, A\&A, accepted

McMahon, R. G., Walton, N. A., Irwin, M. J., et al. 2001, New AR, 45, 97

Meyer, M. R., Calvet, N., \& Hillenbrand, L. A. 1997, ApJ, 114, 288

Meyer, M. R. 2009, IAUS, 258, 111

Merín, B., Jorgensen, J., Spezzi, L., et al. 2008, ApJS, 177, 551

Monet, D. G., Levine, S. E., Canzian, B., et al. 2003, AJ, 125, 984

Oliveira, I., Merín B., Pontoppidan, K. M., et al. 2009, ApJ, 691, 672

Pascucci, I., Apai, D., Luhman, K., et al. 2009, ApJ, 696, 143

Spezzi, L., Alcalà, J. M., Frasca, A., et al. 2007, A\&A, 470, 281

Stetson, P. B. 1987, PASP, 99, 191

Stetson, P. B. 2000, PASP, 112, 925

Straizys, V., Cernis, K., \& Bartasiute, S. 1996, Baltic Astron., 5, 125

Skrutskie, M. F., Cutri, R. M., Stiening, R., et al. 2006, AJ, 131, 1163

Wainscoat, R. J., Cohen, M., Volk, K., et al. 1992, ApJS, 83, 111

Weingartner, J. C., \& Draine, B. T. 2001, ApJ, 548, 296 